\title{
Parametric codebook design for efficient signal transmission using uniform circular arrays
}

\author{
Junsik Shin, Junyeub Suh, Sangchun Park and Wonjin Sung ${ }^{*}$ (D)
}

\section{*Correspondence:}

wsung@sogang.ac.kr Department of Electronic

Engineering, Sogang

University, 35 Baekbeom-ro,

Mapo-gu, Seoul 04107, Korea

\begin{abstract}
In order to improve the quality of the received signal and system spectral efficiency, accurate beamforming using a given antenna array is essential for multiple-input multiple-output (MIMO) systems. To obtain desired MIMO transmission performance, construction of codebooks which are composed of matching beamforming vectors to the array structure is important. To effectively cover different types of mobile traffic, the base station for $5 \mathrm{G}$ new radio employs antenna arrays in various sizes and shapes. Nevertheless, the codebooks adopted by the 3GPP standard so far are based on the uniform linear array and the uniform planar array, necessitating design techniques for a wider class of antenna arrays. In this paper, we propose codebook construction methods for the uniform circular array with parameters to flexibly set the initial phase and step size based on the channel characteristics of the user equipment (UE). When tested over the 3GPP spatial channel model, the proposed codebooks show a substantial amount of gain over the conventional codebooks in all UE locations within the cell.
\end{abstract}

Keywords: MIMO, Codebook, Beamforming, 5G NR, Uniform circular array

\section{Introduction}

To meet the ever-increasing demand for higher data rates, mobile wireless systems including the $5 \mathrm{G}$ new radio (NR) aim to provide the enhanced capacity and coverage to users. One of the key enabling techniques for such an objective is the efficient utilization of multiple-input multiple-output (MIMO) transmission using large-scale arrays [1-4]. To obtain the desired level of performance using MIMO transmission, appropriate beamforming needs to be performed at the transmitter, and pre-designed codebooks are required for practical systems employing the limited feedback for the channel state information (CSI) [5].

The 3GPP standard specifies the codebooks used in multi-antenna transmission. A traditional codebook was designed using the Householder matrix to maximize the spatial distance among codevectors, to support up to 4 transmit antennas [6]. Later versions of the 3GPP codebooks are based on the discrete Fourier transform (DFT) matrix, which work well in spatially correlated channels with a larger number of antennas. These codebooks can also be applied to the cross-polarized antenna arrays with a straightforward extension [7-9]. In general, codevectors optimized for given channel samples can be

(c) The Author(s), 2021. Open Access This article is licensed under a Creative Commons Attribution 4.0 International License, which permits use, sharing, adaptation, distribution and reproduction in any medium or format, as long as you give appropriate credit to the original author(s) and the source, provide a link to the Creative Commons licence, and indicate if changes were made. The images or other third party material in this article are included in the article's Creative Commons licence, unless indicated otherwise in a credit line to the material. If material is not included in the article's Creative Commons licence and your intended use is not permitted by statutory regulation or exceeds the permitted use, you will need to obtain permission directly from the copyright holder. To view a copy of this licence, visit http:// creativecommons.org/licenses/by/4.0/. 
constructed using the vector quantization (VQ) based on the Lloyd-Max algorithm [10]. VQ codebooks are not preferred in practical applications due to its search complexity, but their performance can be used as an upperbound to the performance of other codebooks.

Codebooks adopted in the 3GPP standard assume transmission antenna elements arranged in the uniform linear array (ULA). It is expected, however, that antenna arrays in various sizes and shapes will put into use for enhanced coverage of mobile traffic. For example, small cells can be deployed using street light poles to cope with growing data traffic utilizing existing infrastructure [11]. Antenna arrays other than the ULA may be more suited to such transmission environments, and the uniform circular array (UCA) is a good candidate for installation around pole-type structures. Study results have been reported regarding beamforming methods and related optimization issues for UCAs. In order to steer the main lobe and reduce the side lobe level, sequential quadratic programming is applied in [12] and beam adaptation techniques to changing transmission conditions are studied in [13-15]. To avoid the computational complexity required for the optimization procedure and to apply beamforming developed for the ULA, a transformation method has been suggested to convert the UCA into a virtual ULA [16]. Predesigned codebooks for ULAs can also be applied to circular arrays with appropriate transformation methods. In [17], unitary matrices for the 3GPP Release 8 codebook conversion have been proposed with the analysis of the user equipment (UE) operation. The Hadamard transformation-based codebook design for circular arrays with a reduced feedback mechanism is introduced in [18]. More recently, precoding using the phase-mode transformation for hybrid beamforming using the UCA is investigated for multi-user massive array systems [19]. Increasing cases of UCA utilization can be found in different applications, including transmission over the millimeter-wave channel combined with angle detection [20], non-orthogonal spatial multiplexing transmission [21, 22], and two-dimensional orbital angular momentum (OAM)-based radar imaging [23]. Work by Jing [24] discusses a channel-independent beamforming method over the lineof-sight (LoS) channel using two parallel UCAs at both the transmitter and the receiver. Investigation in [25] applies a low-complexity deep-learning-based DOA estimation scheme to hybrid massive MIMO systems with UCAs. Hybrid beamforming using the UCA is also evaluated and compared with other array structures in [26], showing its advantages in both the sum-rate performance and dominant eigenvalue characteristics. Despite their effectiveness to easily apply existing codebooks to UCAs, many of the earlier works rely on transformed techniques [16-19] which exhibit limitations in beamforming performance. This provides a strong motivation to design a codebook customized to a given circular array structure and channel conditions.

In this paper, we propose a design method for codebooks applicable to circular array structures. While the resulting codevectors retain the DFT property suited to correlated channels, additional customizations are included to reflect the curvature shape of UCAs into the codebook. In addition to its capability to adapt to the amount of the array curvature, the codebook is fully parameterized to flexibly adjust the resolution of codevectors and the distance between them. A parametric codebook design has been attempted for the ULA in [27], and the presented result here is a more in-depth generalization with the additional curvature parameter for the UCA. Furthermore, 
the proposed codebooks are extendable to arbitrary array sizes and channel feedback amounts, making them applicable to massive MIMO applications with varying complexity. The codebook parameters are chosen based on statistical channel characteristics for enhanced beamforming performance for given locations of the target UEs. Performance evaluation results are given for the co-polarized UCAs as well as the cross-polarized extensions. The proposed method here does not involve intensive computational requirements as in [12-15] and can be used in a wide range of applications described in [20-23]. The method can be combined with DOA estimation techniques in [28-30] as well as with an additional element arrangement algorithm [31]. The presented vector parametrization approach can also be applied to important research results in applied sciences, including the antenna design based on fractals [32-39].

The paper is organized as follows. In Sect. 2, the system model and the performance measure are explained, followed by the description of the target array structure. The channel model and its characteristics are analyzed, providing the motivation and guideline for the codebook design. Codebook design examples and their generalization for a wider range of applications are also described, with the definition of proposed codebooks. In Sect. 3, the performance of the proposed codebooks is evaluated and compared with other existing codebooks. Conclusions are given in Sect. 4.

\section{Methods/experimental}

\subsection{System setting}

We consider a downlink MIMO system with the UCA at the base station (BS) and $K$ single-antenna UEs uniformly distributed within the cell as shown in Fig. 1 . The received signal is modeled as

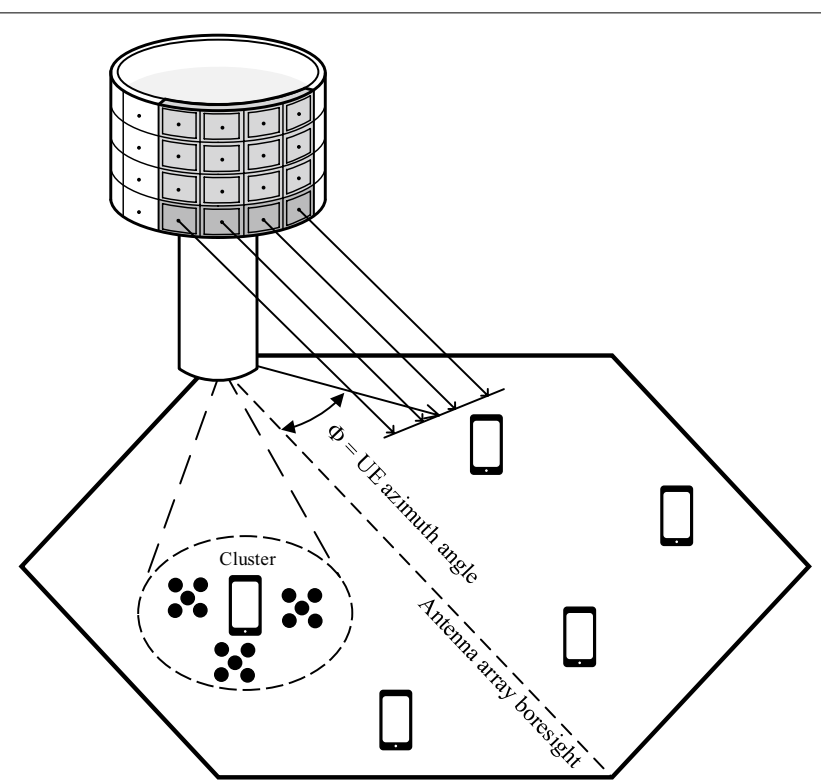

Fig. 1 A cellular transmission model using the uniform circular array 


$$
\mathbf{y}=\mathbf{H W s}+\mathbf{n}
$$

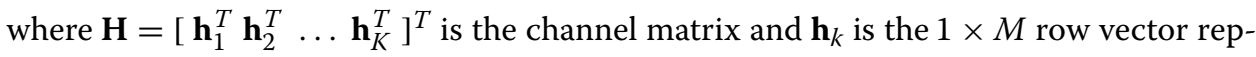
resenting the channel for the $k$-th UE. Precoder matrix $\mathbf{W}=\left[\mathbf{w}_{1} \mathbf{w}_{2} \ldots \mathbf{w}_{K}\right]$ includes the beamforming vector $\mathbf{w}_{k}$ for the $k$-th UE, and $\mathbf{s}$ is the data symbol vector. Each element in noise vector $\mathbf{n}$ follows the complex Gaussian distribution with variance $\sigma^{2}$. Beamforming vectors are chosen from the set of codevectors

$$
\mathbf{C}=\left\{\mathbf{c}_{0}, \mathbf{c}_{1}, \ldots, \mathbf{c}_{Q-1}\right\}
$$

which is the codebook of size $Q$. The codebook size $Q$ and the number of feedback bits $B$ satisfy the relation $Q=2^{B}$. There can be several different criteria in selecting the codevector for the given channel. We adopt the measure to maximize the correlation between the codevector and the channel, i.e., the codevector for the $k$-th UE is chosen as

$$
\mathbf{w}_{k}=\underset{\mathbf{c}_{q} \in \mathbf{C}}{\operatorname{argmax}}\left\|\mathbf{h}_{k} \mathbf{c}_{q}\right\|, q=0,1, \ldots, Q-1
$$

Each UE selects the codevector to maximize its correlation to the channel, and the performance metric of the codebook is the normalized average correlation $\mu$, defined by

$$
\mu=\mathbb{E}\left[\left\|\overline{\mathbf{h}}_{k} \mathbf{w}_{k}\right\|\right]
$$

where $\overline{\mathbf{h}}=\mathbf{h} /\|\mathbf{h}\|$ is the channel with power normalization and $\mathbb{E}[\cdot]$ is the expectation operator. The performance metric has the maximum value of $\mu=1$.

Although a two-dimensional cylinder-type array can be used at the BS such as the one illustrated in Fig. 2, we analyze the channel characteristics to design the corresponding codebook by focusing on the dark shaded area in the figure, i.e., $M$ antenna elements

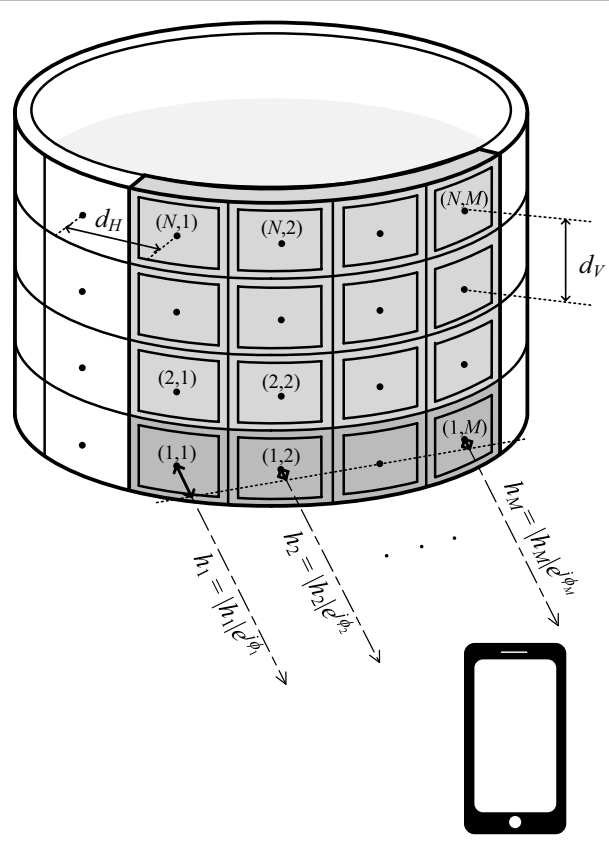

Fig. $2 M$ antennas on a curvature for transmission to the target UE 
located on a curvature. By investigating how channel characteristics behave for the array on curved surface, beamforming vectors can be generated accordingly. For the purpose of brevity, a specific codebook construction will be explained for the case of $M=4$. However, the result can be extended to a general value of $M$ covering any range of azimuth angles including the whole UCA.

Beamforming vector design depends on the shape of the array, since the relative propagation distance for each antenna element differs for a given geometry of the array. Figure 3 represents the propagation distance differences among antenna elements for two different array structures. For the ULA in Fig. 3a, the relative distance linearly increases as the antenna index increases, and so is the relative phase. The extra propagation distance for the $m$-th antenna, when compared to the reference antenna with the shortest propagation distance, is determined as

$$
\xi_{m}=(m-1) d \sin \Phi
$$

for $m=1, \ldots, M$ where $d$ denotes the spacing between adjacent antennas and $\Phi$ is the incidence angle of the signal beam. The phase term caused by the relative propagation distance is

$$
\phi_{m}=2 \pi f_{c} \tau_{m}=\frac{2 \pi}{\lambda} \xi_{m}=\frac{2 \pi}{\lambda}(m-1) d \sin \Phi
$$

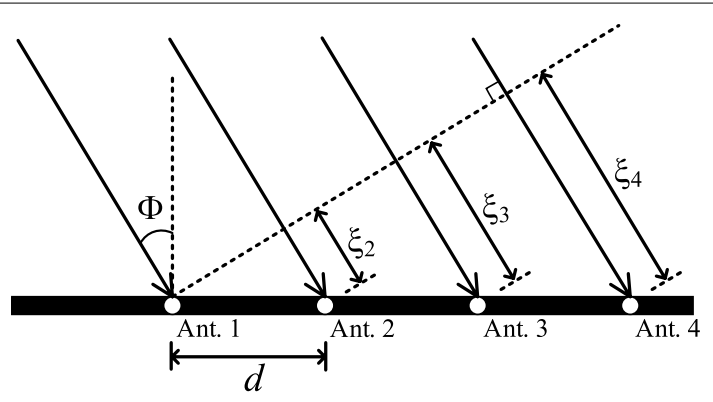

(a)

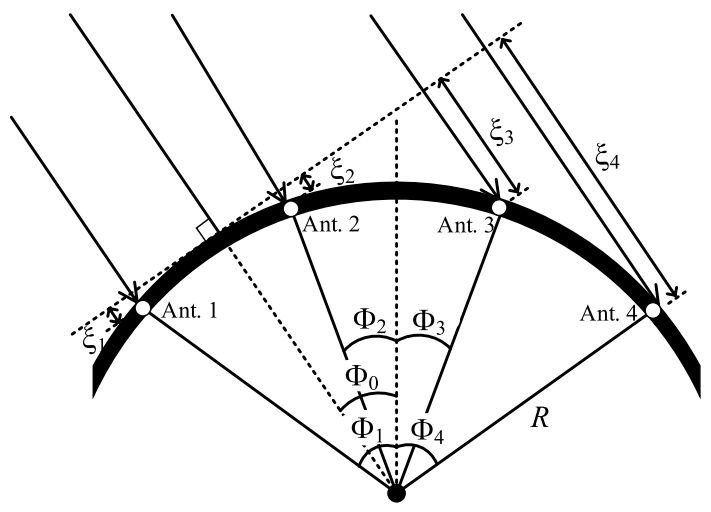

(b)

Fig. 3 Relative propagation distance for each antenna element: a ULA, b UCA 
where $\tau_{m}$ is the relative arrival time difference, $\lambda$ is the carrier wavelength, and $f_{c}$ is the carrier frequency. The LoS-only channel is immediately obtained from the phase terms as

$$
\mathbf{h}_{\mathrm{LoS}}=\left[h_{1} h_{2} \ldots h_{M}\right]=\left[e^{j \phi_{1}} e^{j \phi_{2}} \ldots e^{j \phi_{M}}\right]
$$

and the corresponding beamforming vector can be determined by taking the hermitian of the channel vector as

$$
\mathbf{w}_{\mathrm{ULA}}=\mathbf{h}_{\mathrm{LoS}}^{H}=\left[e^{-j \phi_{1}} e^{-j \phi_{2}} \ldots e^{-j \phi_{M}}\right]^{T} .
$$

It is well known that this type of linearly proportional phase terms for the ULA can be compensated by the vectors taken from the DFT matrix $[5,27]$ and widely adopted codebooks are designed accordingly. For the case of the UCA, the relative propagation distance is not in a linear form as shown in Fig. 3b. As analyzed in [40], the extra distance for the $m$-th antenna in this case is given by

$$
\xi_{m}=R \cos \left(\Phi_{m}-\Phi\right)
$$

for $m=1, \ldots, M$, where $R$ is the radius of the UCA and $\Phi_{m}$ is the azimuth position angle for the $m$-th antenna element. The phase is determined as

$$
\phi_{m}=\frac{2 \pi}{\lambda} R \cos \left(\Phi_{m}-\Phi\right)
$$

and the beamforming vector for the UCA is obtained as

$$
\mathbf{w}_{\mathrm{UCA}}=\left[e^{-j \phi_{1}} e^{-j \phi_{2}} \ldots e^{-j \phi_{M}}\right]^{T} .
$$

While the beamforming vector in (11) exactly compensates for the relative phase values caused by the UCA geometry, it has the infinite resolution and cannot be immediately utilized as the candidate codevector for the finite feedback channel. Furthermore, practical wireless channels quite often include a number of multipath components, and the codebook based on the LoS channel only may not provide desired beamforming performance. To overcome these issues and to find an effective design strategy for the codebook construction, we investigate channel properties for the UCA.

\subsection{Channel characteristics experiment}

Modeling the wireless channel is important not only to accurately estimate the transmission performance but to appropriately design signal processing strategies at both the transmitter and receiver. 3GPP adopts the spatial channel model (SCM) [41] for the standardization and evaluation of signaling methods. The model includes multiple clusters distributed over the three-dimensional space such that the resulting multipath channel resembles the wireless link of the real-world environment as much as possible. SCM is especially useful in verification of MIMO beamforming performance, since channel coefficients of the antenna elements are generated with detailed probabilistic procedures reflecting the location and geometry of the array. In order to present a practical codebook construction result which can potentially be utilized as a radio access standard, we apply the SCM for the generation of channel coefficients 
in our signal model (1) and use the urban macro (UMa) scenario for the design and evaluation of the proposed codebook.

Figure 4 shows the array shapes for the ULA and UCA, where $h_{m}$ denotes the complex channel coefficient for the $m$-th antenna element. Let $\phi_{m}$ denote the phase component of $h_{m}$, then the phase difference between adjacent elements is expressed as

$$
\theta_{m}=\phi_{m+1}-\phi_{m}
$$

for $m=1, \ldots, M-1$. Figure 5 shows the representation of the probability density function (PDF) for $\theta_{m}$, obtained by repeated generations of channel coefficients for the target UE located at the azimuth angle of $\Phi=30^{\circ}$ using the SCM simulator. Half-wavelengths spacing is applied for the distance between adjacent antenna elements for both arrays. For the ULA of $M=4$ antenna elements, the PDFs for $\theta_{1}, \theta_{2}$ and $\theta_{3}$ shown in Fig. 5a are almost identical. This is an expected result because the relative distance between adjacent elements is the same regardless of the antenna index. On the other hand, the PDFs for the UCA in Fig. 5b are clearly distinguishable from one another, which is due to the difference in relative distances of propagation paths. Geometric characteristics of the circular array resulted in channel distributions that are quite different from ULA distributions. Figure 5b shows that the PDF curves are approximately shifted versions of one another. This observation leads to adding an extra parameter to the codevector design for the UCA, to compensate for the amount of shifts experienced in channel distributions.

The phase difference in (12) can be determined using the phase term in (6) for the ULA as

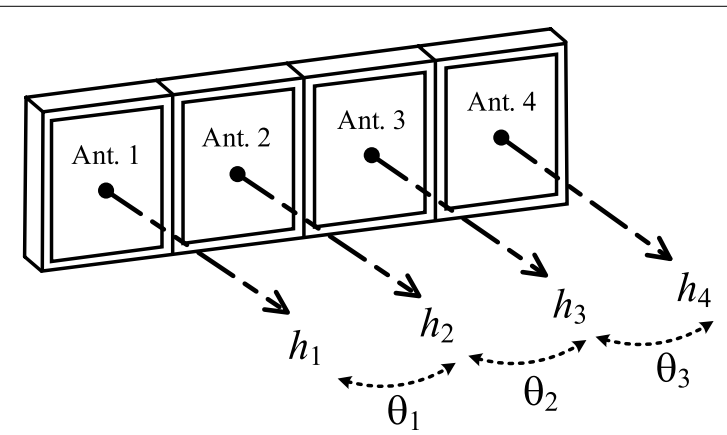

(a)

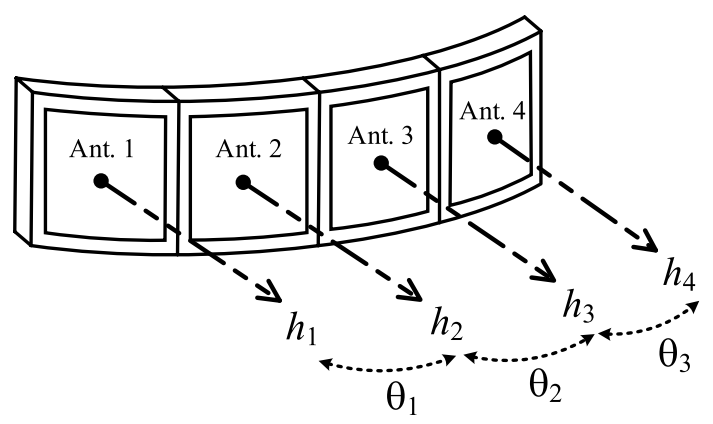

(b)

Fig. 4 Phase difference between adjacent antenna elements: a ULA, b UCA 


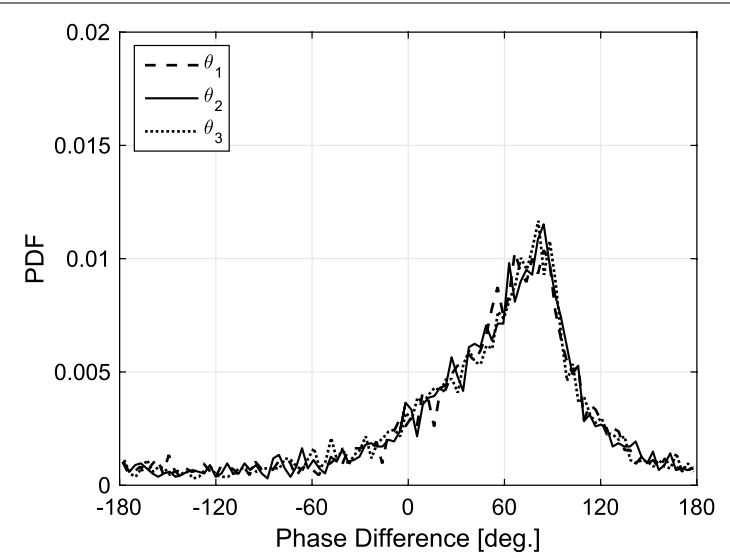

(a)

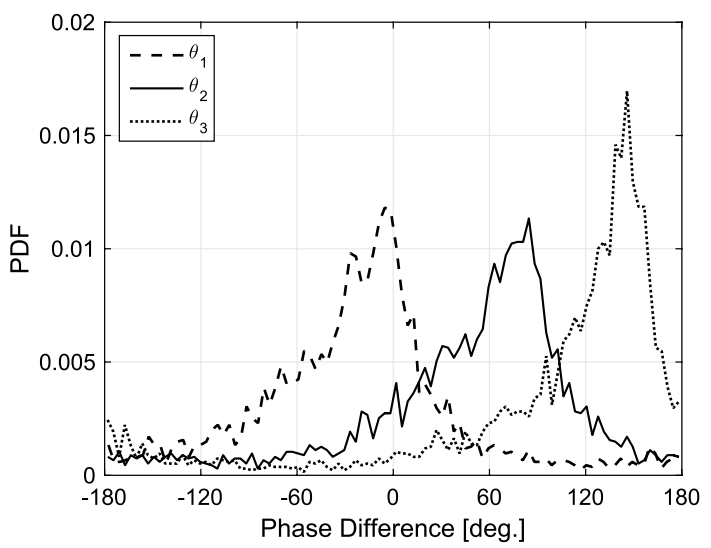

(b)

Fig. 5 Distributions of the phase difference between adjacent antenna elements at $\Phi=30^{\circ}$ : a ULA, $\mathbf{b} \cup C A$

$$
\theta_{m}=\frac{2 \pi}{\lambda} d \sin \Phi
$$

which simplifies to $\theta_{m}=\pi \sin \Phi$ when $d=\lambda / 2$. While the user azimuth angle $\Phi$ is a fixed value for the given location, SCM generates a number of propagation rays with incidence angles centered around $\Phi$. Thus, the median value of the probabilistic phase difference distribution can be approximated to $\pi \sin \Phi$. We have $\pi \sin (\pi / 6)=\pi / 2$ when $\Phi=30^{\circ}$, which is very close to the actual median value $87^{\circ}$ of $\theta_{m}$ observed in the distribution shown in Fig. 5a. For the UCA, the phase difference depends on antenna index $m$ and a simplified expression for the median value is not immediately obtainable. However, we observe that the overall shape of the PDF is similar for all $m$ with a certain amount of shift in distributions in Fig. 5b. Therefore, an approximated relation for the phase difference can be expressed as

$$
\theta_{m+1}=\theta_{m}+\chi
$$

where $\chi$ is the additional parameter introduced in the proposed codebook design. We describe the parametric codebook for the ULA fist, followed by the codebook construction methods for the UCA using additional parameters in the next section. Construction 
examples are given for the case of $M=4$, which are extended to general expressions for any given number of antenna elements.

\subsection{Design methods for parametric codebooks}

For the ULA with linear change in phase values, a basic form of codevectors can be expressed as

$$
\mathbf{c}=\frac{1}{\sqrt{M}}\left[1 e^{-j \gamma} \ldots e^{-j(M-1) \gamma}\right]^{T}
$$

where $\gamma$ is adjusted to compensated for the phase difference between adjacent antenna elements. For $M=4$ antennas, the expression reduces to

$$
\mathbf{c}=\frac{1}{2}\left[1 e^{-j \gamma} e^{-j 2 \gamma} e^{-j 3 \gamma}\right]^{T} .
$$

In a codebook including $Q$ codevectors, we can choose $Q$ different values of $\gamma$ in such a way that these values effectively cover the distribution of the phase difference $\theta_{m}$. Let us consider a construction example for the codebook of size $Q=4$. In Fig. 6, four cross-marks indicate $\gamma$ values to be used in four codevectors. Setting $\gamma=\alpha$ gives the first codevector $\mathbf{c}_{0}=(1 / 2)\left[1 e^{-j \alpha} e^{-j 2 \alpha} e^{-j 3 \alpha}\right]^{T}$, where $\alpha$ is referred to as the initial phase parameter. The second codevector uses $\gamma=\alpha+\beta$ to become $\mathbf{c}_{1}=(1 / 2)\left[1 e^{-j(\alpha+\beta)} e^{-j 2(\alpha+\beta)} e^{-j 3(\alpha+\beta)}\right]^{T}$, where $\beta$ is called the step size parameter. The third codevectors is obtained by increasing $\gamma$ in the amount of step size $\beta$ to set $\gamma=\alpha+2 \beta$. Likewise, the fourth codevector uses $\gamma=\alpha+3 \beta$. Including these column codevectors in a matrix gives the codevector matrix for the ULA

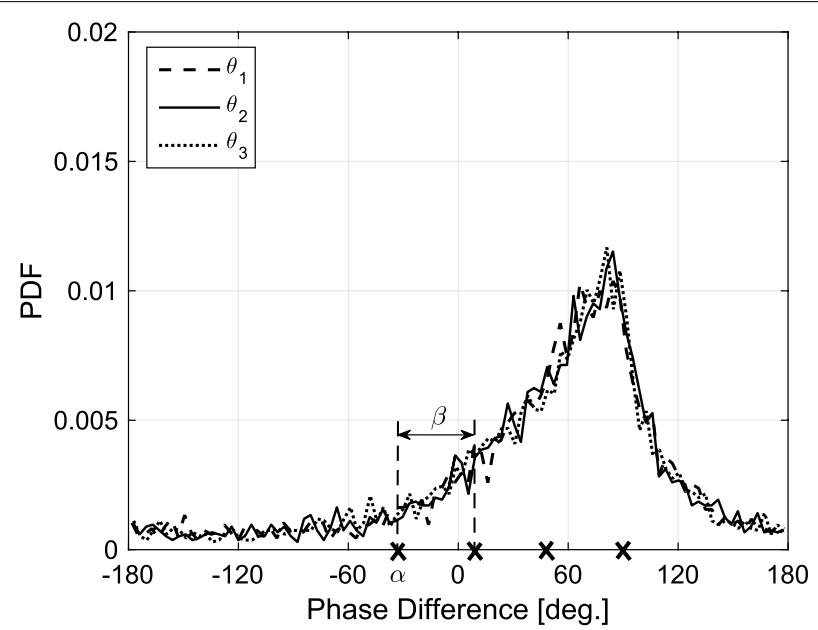

Fig. 6 Parametric codebook design for the ULA using parameters $\alpha$ and $\beta$ 


$$
\begin{aligned}
\mathbf{C}_{\mathrm{ULA}} & =\left[\mathbf{c}_{0} \mathbf{c}_{1} \mathbf{c}_{2} \mathbf{c}_{3}\right] \\
& =\frac{1}{2}\left[\begin{array}{cccc}
1 & 1 & 1 & 1 \\
e^{-j \alpha} & e^{-j(\alpha+\beta)} & e^{-j(\alpha+2 \beta)} & e^{-j(\alpha+3 \beta)} \\
e^{-j 2 \alpha} & e^{-j 2(\alpha+\beta)} & e^{-j 2(\alpha+2 \beta)} & e^{-j 2(\alpha+3 \beta)} \\
e^{-j 3 \alpha} & e^{-j 3(\alpha+\beta)} & e^{-j 3(\alpha+2 \beta)} & e^{-j 3(\alpha+3 \beta)}
\end{array}\right]
\end{aligned}
$$

which is a $4 \times 4$ matrix. The number of rows represents the number of antennas, and the number of columns is the codebook size. Therefore, in general, we obtain an $M \times Q$ codebook matrix. The codebook given in (17) is the long-term codebook for a given UE location, from which a specific codevector is chosen based on the short-term channel state [5]. This is similar to the 3GPP long-term codebook, which consists of 4 consecutive codevectors out of 32 DFT beams over all directions as specified in [7]. A more detailed description of the parametric codebook for the ULA can be found in [27], which gives the general construction procedure as well as the performance evaluation result.

We further generalize the parametric codebook construction result for the ULA to the case of the UCA. In order to compensate for the distribution shifts for the phase difference, the curvature parameter $\chi$ is used as illustrated in Fig. 7. Using (14), we obtain $\theta_{2}=\theta_{1}+\chi$ and $\theta_{3}=\theta_{2}+\chi=\theta_{1}+2 \chi$. When we set $\theta_{1}=\gamma$ for the phase term compensation, it follows that

$$
\begin{aligned}
& \phi_{2}=\phi_{1}+\theta_{1}=\phi_{1}+\gamma \\
& \phi_{3}=\phi_{2}+\theta_{2}=\left(\phi_{1}+\gamma\right)+(\gamma+\chi)=\phi_{1}+2 \gamma+\chi \text { and } \\
& \phi_{4}=\phi_{3}+\theta_{3}=\left(\phi_{1}+2 \gamma+\chi\right)+(\gamma+2 \chi)=\phi_{1}+3 \gamma+3 \chi
\end{aligned}
$$

using the relation given in (12). Since only the relative phase values affect the beamforming characteristics, common phase term $\phi_{1}$ can be removed from all elements in codevectors. Thus, we obtain the UCA codebook matrix including four column codevectors with respective phase compensation terms $\gamma=\alpha, \alpha+\beta, \alpha+2 \beta$ and $\alpha+3 \beta$ as

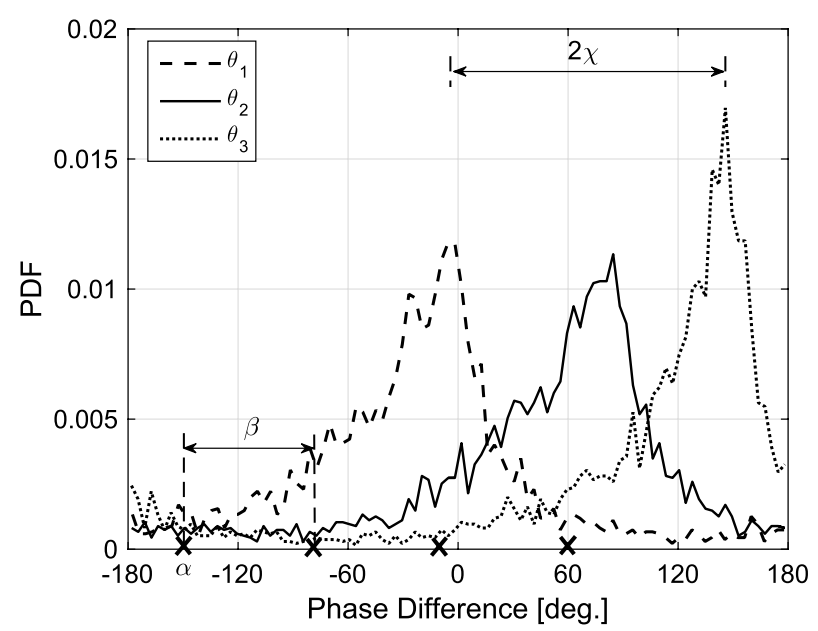

Fig. 7 Parametric codebook design for the UCA using the curvature parameter $\chi$ 
Table 1 Parameters for $\mathbf{C}_{U C A, 1}$ maximizing the average correlation in the SCM UMa environment

\begin{tabular}{llllll}
\hline$\Phi$ & $\mathbf{0}^{\circ}$ & $\mathbf{1 5 ^ { \circ }}$ & $\mathbf{3 0 ^ { \circ }}$ & $\mathbf{4 5 ^ { \circ }}$ & $\mathbf{6 0 ^ { \circ }}$ \\
\hline$\alpha$ & $-158^{\circ}$ & $-182^{\circ}$ & $-148^{\circ}$ & $-122^{\circ}$ & $-84^{\circ}$ \\
$\beta$ & $66^{\circ}$ & $68^{\circ}$ & $70^{\circ}$ & $76^{\circ}$ & $78^{\circ}$ \\
$\chi$ & $90^{\circ}$ & $86^{\circ}$ & $78^{\circ}$ & $72^{\circ}$ & $58^{\circ}$ \\
\hline
\end{tabular}

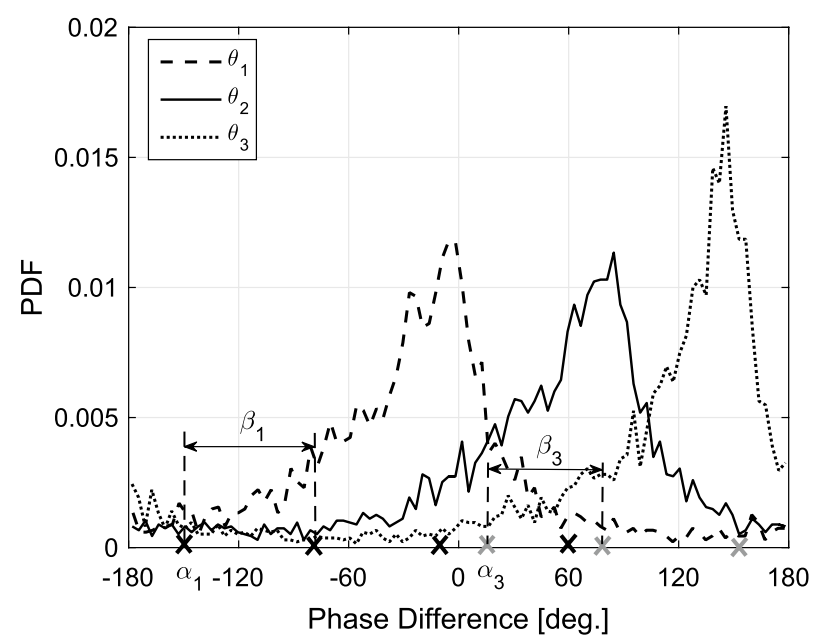

Fig. 8 Parametric codebook design for the UCA using multiple parameter pairs $\left(\alpha_{k}, \beta_{k}\right)$

$$
\begin{aligned}
\mathbf{C}_{\mathrm{UCA}, 1} & =\left[\mathbf{c}_{0} \mathbf{c}_{1} \mathbf{c}_{2} \mathbf{c}_{3}\right] \\
& =\frac{1}{2}\left[\begin{array}{cccc}
1 & 1 & 1 & 1 \\
e^{-j \alpha} & e^{-j(\alpha+\beta)} & e^{-j(\alpha+2 \beta)} & e^{-j(\alpha+3 \beta)} \\
e^{-j\{2 \alpha+\chi\}} & e^{-j\{2(\alpha+\beta)+\chi\}} & e^{-j\{2(\alpha+2 \beta)+\chi\}} & e^{-j\{2(\alpha+3 \beta)+\chi\}} \\
e^{-j\{3 \alpha+3 \chi\}} & e^{-j\{3(\alpha+\beta)+3 \chi\}} & e^{-j\{3(\alpha+2 \beta)+3 \chi\}} & e^{-j\{3(\alpha+3 \beta)+3 \chi\}}
\end{array}\right] .
\end{aligned}
$$

By comparing two codebooks in (17) and (20), we note that additional compensation terms $\chi$ and $3 \chi$ exist for the third and fourth element in codevectors for the UCA, as explained in relations given by (18) and (19). Codebook $\mathbf{C}_{\mathrm{UCA}, 1}$ in (20) is the proposed codebook for the UCA of the first kind. Specific parameter values can be determined based on actual channel coefficients generated for the given environment. Table 1 shows the codebook parameters which maximizes the average correlation in (4) at different user azimuth angles. Channel coefficients are repeatedly generated using the SCM UMa simulator at user locations $\Phi=0,15,30,45$, and 60 degrees, and the optimizing parameters for the codebook of size $Q=4$ are selected. The parameters can also be determined for negative azimuth angles using the symmetry of the channel distributions.

Since the distributions for $\theta_{1}, \theta_{2}$, and $\theta_{3}$ are not exactly the same, separate parameters can be used for each of these distributions. As shown in Fig. 8, parameter pair $\left(\alpha_{k}, \beta_{k}\right)$ is used for the distribution of $\theta_{k}$, for $k=1,2$, and 3 . In the proposed codebook for the UCA of the second kind, the first codevector is chosen as

$$
\mathbf{c}_{0}=\frac{1}{2}\left[1 e^{-j \alpha_{1}} e^{-j\left(\alpha_{1}+\alpha_{2}\right)} e^{-j\left(\alpha_{1}+\alpha_{2}+\alpha_{3}\right)}\right]^{T}
$$


and the second codevector is determined as

$$
\mathbf{c}_{1}=\frac{1}{2}\left[1 e^{-j\left(\alpha_{1}+\beta_{1}\right)} e^{-j\left\{\left(\alpha_{1}+\beta_{1}\right)+\left(\alpha_{2}+\beta_{2}\right)\right\}} e^{-j\left\{\left(\alpha_{1}+\beta_{1}\right)+\left(\alpha_{2}+\beta_{2}\right)+\left(\alpha_{3}+\beta_{3}\right)\right\}}\right]^{T} .
$$

Continuing this construction for the remaining two codevectors, the codebook matrix becomes

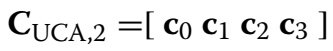

$$
\begin{aligned}
& =\frac{1}{2}\left[\begin{array}{cccc}
1 & 1 & 1 & 1 \\
e^{-j \sum_{k=1}^{1} \alpha_{k}} & e^{-j \sum_{k=1}^{1}\left(\alpha_{k}+\beta_{k}\right)} & e^{-j \sum_{k=1}^{1}\left(\alpha_{k}+2 \beta_{k}\right)} & e^{-j \sum_{k=1}^{1}\left(\alpha_{k}+3 \beta_{k}\right)} \\
e^{-j \sum_{k=1}^{2} \alpha_{k}} & e^{-j \sum_{k=1}^{2}\left(\alpha_{k}+\beta_{k}\right)} & e^{-j \sum_{k=1}^{2}\left(\alpha_{k}+2 \beta_{k}\right)} & e^{-j \sum_{k=1}^{2}\left(\alpha_{k}+3 \beta_{k}\right)} \\
e^{-j \sum_{k=1}^{3} \alpha_{k}} & e^{-j \sum_{k=1}^{3}\left(\alpha_{k}+\beta_{k}\right)} & e^{-j \sum_{k=1}^{3}\left(\alpha_{k}+2 \beta_{k}\right)} & e^{-j \sum_{k=1}^{3}\left(\alpha_{k}+3 \beta_{k}\right)}
\end{array}\right] .
\end{aligned}
$$

Parameters maximizing the average correlation are also found for the codebook of the second kind, and their numeric values are shown in Table 2 for different user azimuth angles.

Proposed codebooks can be generalized to arbitrary numbers of antenna elements and feedback bits. Suppose we construct a codebook of size $Q=2^{B}$ using $B$ feedback bits for $M$ antenna elements. The $M \times Q$ codebook matrix is denoted by

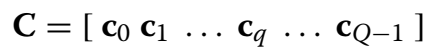

where the $q$-th codevector can be written as

$$
\mathbf{c}_{q}=\left[\begin{array}{llllll}
c_{0, q} & c_{1, q} & \ldots & c_{m, q} & \ldots & c_{M-1, q}
\end{array}\right]^{T}
$$

for $q=0,1, \ldots, Q-1$. For the proposed codebook of the first kind, element $c_{m, q}$ is determined as

$$
c_{m, q}=\frac{1}{\sqrt{M}} e^{-j\left\{m(\alpha+q \beta)+F_{m} \chi\right\}}
$$

for $m=0,1, \ldots, M-1$, where we defined $F_{m}=\sum_{k=1}^{m-1} k$. Thus, the $q$-th codevector can be written as

Table 2 Parameters for $\mathbf{C}_{U C A, 2}$ maximizing the average correlation in the SCM UMa environment

\begin{tabular}{llllll}
\hline$\Phi$ & $\mathbf{0}^{\circ}$ & $\mathbf{1 5 ^ { \circ }}$ & $\mathbf{3 0 ^ { \circ }}$ & $\mathbf{4 5 ^ { \circ }}$ & $\mathbf{6 0 ^ { \circ }}$ \\
\hline$\alpha_{1}$ & $-158^{\circ}$ & $-182^{\circ}$ & $-148^{\circ}$ & $-122^{\circ}$ & $-84^{\circ}$ \\
$\alpha_{2}$ & $-142^{\circ}$ & $-88^{\circ}$ & $-68^{\circ}$ & $-44^{\circ}$ & $-10^{\circ}$ \\
$\alpha_{3}$ & $-46^{\circ}$ & $-12^{\circ}$ & $14^{\circ}$ & $26^{\circ}$ & $26^{\circ}$ \\
$\beta_{1}$ & $66^{\circ}$ & $68^{\circ}$ & $70^{\circ}$ & $76^{\circ}$ & $78^{\circ}$ \\
$\beta_{2}$ & $72^{\circ}$ & $68^{\circ}$ & $74^{\circ}$ & $76^{\circ}$ & $74^{\circ}$ \\
$\beta_{3}$ & $68^{\circ}$ & $66^{\circ}$ & $68^{\circ}$ & $68^{\circ}$ & $70^{\circ}$ \\
\hline
\end{tabular}




$$
\mathbf{c}_{q}=\frac{1}{\sqrt{M}}\left[\begin{array}{c}
1 \\
e^{-j\{\alpha+\beta\}} \\
e^{-j\{2(\alpha+\beta)+\chi\}} \\
\vdots \\
e^{-j\left\{(M-1)(\alpha+\beta)+\sum_{k=1}^{M-2} \chi\right\}}
\end{array}\right] .
$$

For the proposed codebook of the second kind, element $c_{m, q}$ is expressed as

$$
c_{m, q}=\frac{1}{\sqrt{M}} e^{-j \sum_{k=1}^{m}\left(\alpha_{k}+q \beta_{k}\right)}
$$

for $m=0,1, \ldots, M-1$ and we obtain the $q$-th codevector as

$$
\mathbf{c}_{q}=\frac{1}{\sqrt{M}}\left[\begin{array}{c}
1 \\
e^{-j\left(\alpha_{1}+\beta_{1}\right)} \\
e^{-j \sum_{k=1}^{2}\left(\alpha_{k}+\beta_{k}\right)} \\
\vdots \\
e^{-j \sum_{k=1}^{M-1}\left(\alpha_{k}+\beta_{k}\right)}
\end{array}\right] .
$$

Finally, the codevectors can be extended to the cross-polarized (X-pol) antenna arrays as described in [7]. Suppose $\mathbf{c}_{q}$ is a codevector for the co-polarized array of $M$ antenna elements, such as the ones given in (27) and (29). The extended codevector for the crosspolarized array of $2 M$ elements is obtained as

$$
\mathbf{c}_{q}^{\prime}=\left[\begin{array}{c}
\mathbf{c}_{q} \\
\zeta \\
\mathbf{c}_{q}
\end{array}\right]
$$

where $\zeta$ is the co-phasing term which takes one of the values in $\{ \pm 1, \pm j\}$.

\section{Results and discussion}

In order to demonstrate the effectiveness of the proposed codebooks, we evaluate and compare the performance of well-known existing codebooks under the same environmental settings. A traditional 3GPP codebook using the Householder matrix is constructed from the generation vector $\mathbf{u}_{k}$ of length 4 , which contains elements from the 8PSK alphabet $\{ \pm 1, \pm j, \pm(1+j) / \sqrt{2}, \pm(1-j) / \sqrt{2})\}$ as described in [6]. The codevectors are obtained by

$$
\mathbf{a}_{k}=\mathbf{I}-\frac{2 \mathbf{u}_{k} \mathbf{u}_{k}^{H}}{\mathbf{u}_{k}^{H} \mathbf{u}_{k}}
$$

for $k=0,1, \ldots, 15$ where I is the identity matrix. The later versions of the 3GPP codebook are based on the $4 \times 32$ matrix consisting 32 DFT vectors. Beamforming is operated in two stages. In the first stage, 4 consecutive column vectors out of the 32 columns in the matrix are chose as the long-term codebook based on the channel characteristics of the user. In the subsequent stage, the beamforming vector that matches best to the instantaneous channel condition is chosen as the short-term codevector. The long-term codebook consists of 4 consecutive DFT vectors as in 


$$
\mathbf{B}_{k}=\left[\mathbf{b}_{2 k} \mathbf{b}_{2 k+1} \mathbf{b}_{(2 k+2) \bmod 32} \mathbf{b}_{(2 k+3) \bmod 32}\right]
$$

for $k=0,1, \ldots, 15$, where

$$
\mathbf{b}_{q}=\frac{1}{2}\left[1 e^{j \frac{2 \pi}{32} q} e^{j \frac{2 \pi}{32} 2 q} e^{j \frac{2 \pi}{32} 3 q}\right]^{T}
$$

is the DFT codevector. A more general realization of the codebook using the DFT vectors is the uniform DFT codebook covering the entire azimuth directions in uniform beam resolution [5]. The uniform DFT codebook of size $Q$ applied to antenna arrays with $M$ elements is described by $M \times Q$ matrix $\mathbf{C}=\left[\begin{array}{llll}\mathbf{c}_{0} & \mathbf{c}_{1} \ldots \mathbf{c}_{Q-1}\end{array}\right]$ where

$$
\mathbf{c}_{q}=\frac{1}{\sqrt{M}}\left[1 e^{j \frac{2 \pi}{Q} q} e^{j \frac{2 \pi}{Q} 2 q} \ldots, e^{j \frac{2 \pi}{Q}(M-1)}\right]^{T}
$$

for $q=0,1, \ldots, Q-1$. These DFT-based codebooks are primarily targeted to the ULA and thus may not work very well for other types of arrays. There exists a transformation method, however, to convert the DFT codebooks into a form suitable to the UCA. The method proposed in [18] uses an $M \times M$ Hadamard matrix to transform the DFT codebook matrix into a modified codebook for the circular array, which is known to outperform codebooks obtained from other transformation methods. To obtain a performance upperbound, the VQ codebook can be constructed using the Lloyd-Max algorithm based on a sufficient number of training vectors obtained from the target channel environment. By repeatedly applying the nearest neighborhood condition and the centroid condition, converging codevectors are obtained. Despite the near optimal performance, the VQ codebook is not applicable to practical systems due to its huge computational complexity and inadaptability to changing channel conditions. All discussed codebooks can be extended to the cross-polarized arrays using the co-phasing term as described in (30).

Figure 9 shows the location-wise performance of proposed codebooks, compared with those of existing codebooks applied to the UCA of $M=4$ antenna elements. At each

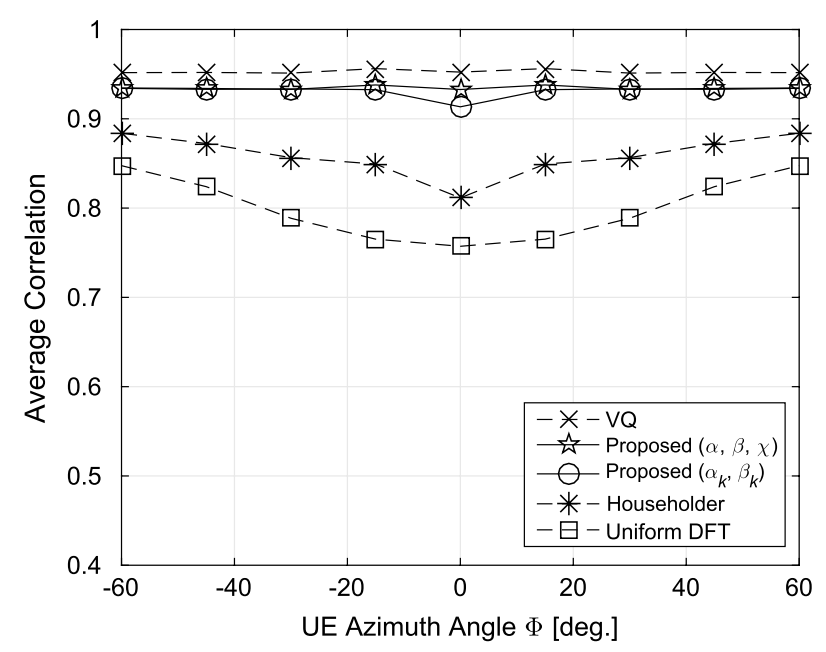

Fig. 9 Location-specific performance of codebooks using $B=4$ feedback bits 
value of the UE azimuth angle, channel coefficients are repeatedly generated to evaluate the average correlation of the best-matching codevector in the given codebook. The proposed UCA codebook of the first kind is indicated by parameters $(\alpha, \beta, \chi)$ in the figure. Similarly, the proposed UCA codebook of the second kind is indicated by the parameter set $\left(\alpha_{k}, \beta_{k}\right)$ which is used for the codebook generation. It can be confirmed from the figure that both of these two types of codebooks perform extremely well over the entire range of user locations, exhibiting the performance close to the VQ codebook found by search. This is in contrast with existing 3GPP codebooks shown together in the figure, showing severe performance degradations. All codebooks in the figure uses the same number of codevectors. Each codebook includes $Q=16$ codevectors using the $B=4$ feedback bits.

Figure 10 shows the performance comparison when different numbers of feedback bits are used. By using $B=2,3$ and 4 bits, the codebook size, respectively, becomes $Q=4,8$ and 16. The UEs are randomly generated within the cell, and the channel coefficients are determined at given locations. Therefore, the figure shows the cell-average performance. For all values of codebook size tested, two kinds of proposed codebooks significantly outperform the existing ones, with the correlation performance close to the upperbound. Some of the comparing codebooks are known for only at certain codebook sizes, e.g., the 3GPP codebook of size 16 using the Householder matrix. It can be observed that the codebook obtained by the Hadamard transformation method, although it is specifically targeted for the UCA, provides a significantly lower correlation performance than proposed codebooks.

The performance evaluation results can be extended to cross-polarized circular arrays with $M=8$ antenna elements. In Fig. 11, location-wise performance is shown by applying four co-phasing terms $\zeta= \pm 1$ and $\pm j$ which require additional 2 bits for the phase information feedback. The correlation values in the figure are obtained by using $Q=4$ codevectors requiring the 2-bit feedback. Combining 2 codevector selection bits and 2 co-phasing bits, the total of 4 feedback bits is used for this operation. The proposed codebooks also outperform all existing codebooks for the cross-polarized array with

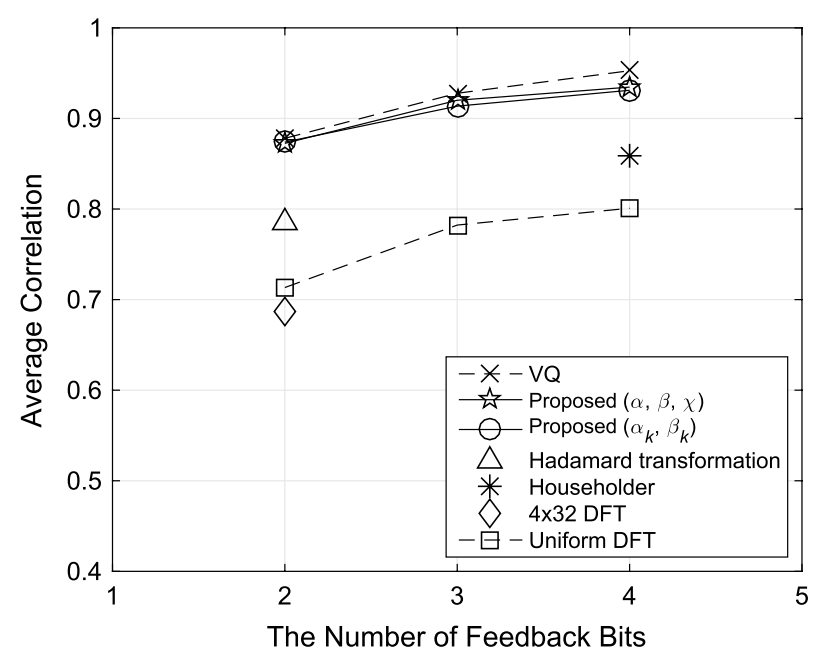

Fig. 10 Cell-average performance of codebooks using $B=2,3$ and 4 feedback bits 


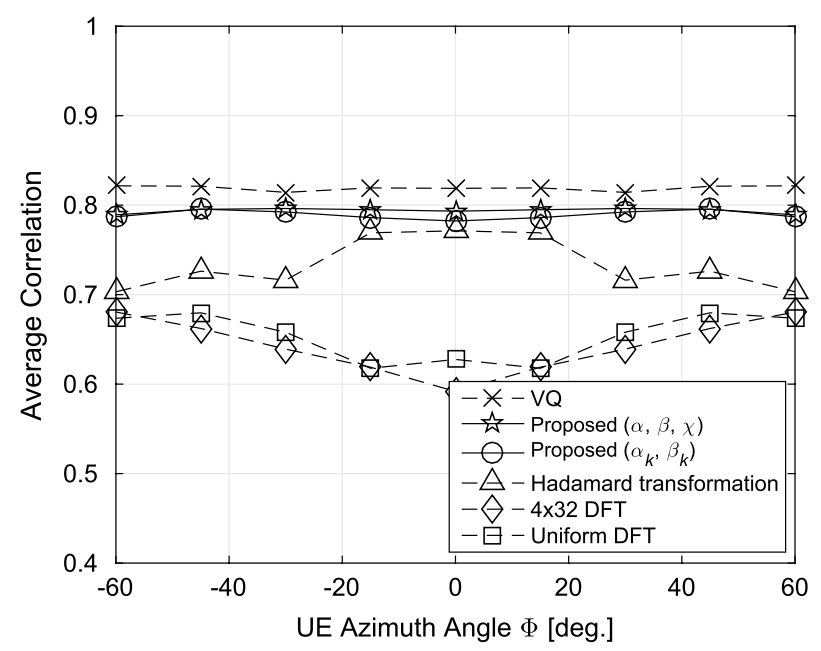

Fig. 11 Location-specific performance of codebooks for the cross-polarized antenna array

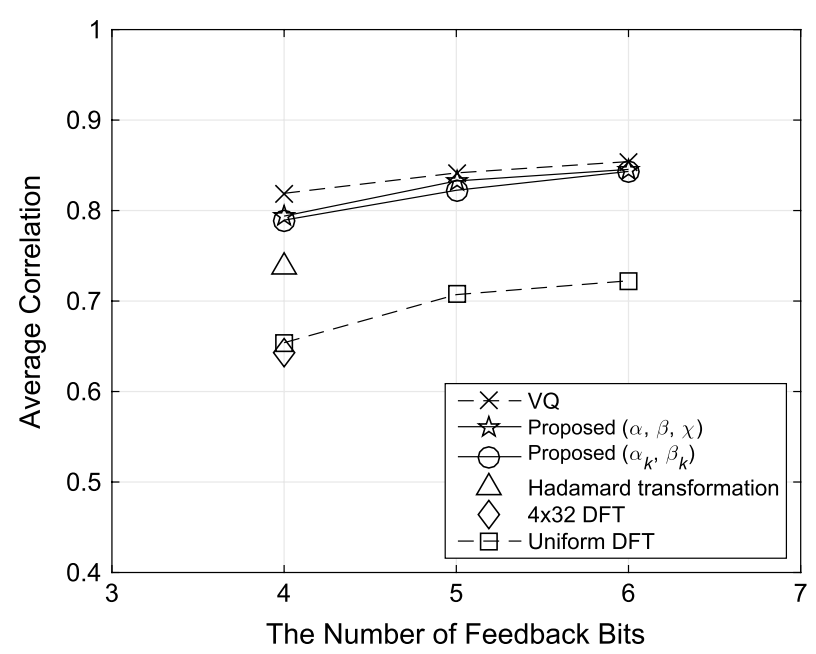

Fig. 12 Cell-average performance of codebooks for the cross-polarized antenna array

substantial margins. The correlation values of the proposed codebooks are uniform over all azimuth angles, suggesting they can be reliably applied for beamforming regardless of the user locations. The advantage of the proposed codebooks for the cross-polarized array is again confirmed for the cell-wise performance in Fig. 12, which indicates a large amount of gains for all numbers of feedback bits. The number of feedback bits 4,5 , and 6 in the $X$-axis of the figure, respectively, correspond to codebook size of 4,8 , and 16 , in addition to 4 co-phasing terms requiring 2 extra feedback bits.

\section{Conclusions}

Codebook construction methods using multiple parameters adjustable to the varying channel distributions for the UCA are proposed. The proposed codebooks can be determined for arbitrary numbers of antenna elements and feedback bits and are applicable to both co-polarized and cross-polarized circular arrays. By evaluating over the 3GPP 
channel model reflecting practical environmental settings, the proposed codebooks are shown to provide enhanced performance over the conventional codebooks.

\section{Abbreviations}

MIMO: Multiple-input multiple-output; BS: Base station; 5G: Fifth generation; NR: New radio; 3GPP: 3rd-generation partnership project; ULA: Uniform linear array; UPA: Uniform planar array; UCA: Uniform circular array; UE: User equipment; SCM: Spatial channel model; CSI: Channel state information; DFT: Discrete Fourier transform; VQ: Vector quantization.

\section{Acknowledgements}

This work was supported by the National Research Foundation (NRF) of Korea, Ministry of Science, ICT and Future Planning (MSIP) under Grant NRF-2020R1A2C1004135.

\section{Authors' contributions}

J. Shin and W. Sung drafted the manuscript. Both authors read and approved the final manuscript.

Availability of data and materials

Data sharing not applicable to this article during the current study.

\section{Declaration}

Competing interests

The authors declare that they have no competing interests.

Received: 28 June 2021 Accepted: 7 November 2021

Published online: 27 November 2021

\section{References}

1. S. Parkvall, E. Dahlman, A. Furuskar, M. Frenne, NR: the new 5 G radio access technology. IEEE Commun. Stand. Mag. 1(4), 24-30 (2017)

2. A. Ghosh, A. Maeder, M. Baker, D. Chandramouli, $5 \mathrm{G}$ evolution: a view on $5 \mathrm{G}$ cellular technology beyond $3 \mathrm{GPP}$ Release 15. IEEE Access 7(1), 127639-127651 (2019)

3. S. Chen, S. Sun, G. Xu, X. Su, Y. Cai, Beam-space multiplexing: practice, theory, and trends, from 4G TD-LTE, 5G, to 6G and beyond. IEEE Wirel. Commun. 27(2), 162-172 (2020)

4. A. Rodriguez-Herrera, D. Reed, J.P. Nuutinen, in Proceedings of the 2021 15th European Conference on Antennas and Propagation (EUCAP), Spatial Channel Model Validation for 3GPP FR2 MIMO OTA (2021)

5. X. Su, J. Zeng, J. Li, Rong, L. Liu, X. Xu, J. Wang, Limited feedback precoding for massive MIMO. Int. J. Antennas Propag. 2013, Article ID 436352 (2013)

6. 3GPP TS 36.211, Evolved Universal Terrestrial Radio Access (E-UTRA); Physical Channels and Modulation, V15.6.0 (2019)

7. 3GPP TS 36.213, E-UTRA; Physical Layer Procedures, V15.6.0 (2019)

8. 3GPP TSG RAN R1-155490, Rank 1-2 Codebook for Class A CSI Reporting (2015)

9. 3GPP TSG RAN R1-157789, Joint Proposal on Rank 2-8 Codebook for Class A and Class B (2015)

10. J. Park, J. Kim, H. Yoo, W. Sung, in Proceedings of the IEEE Wireless Communications and Networking Conference Construction of Phase Tracking Codebooks Based on the Lloyd-Max Vector Quantization (Cancun, Mexico, 2011)

11. Signals Research Group, Street light small cells-A revolution in mobile operator networks economics. White Paper sponsored by InterDigital (2014)

12. A. Hammami, R. Ghayoula, A. Gharsallah, in Proceedings of the IEEE Mediterranean Microwave Symposium, Uniform Circular Phased Arrays Synthesis Using SQP Algorithm (Hammamet, Tunisia, 2011)

13. N.H. Noordin, V. Zuniga, A.O. El-Rayis, N. Haridas, A.T. Ergodan, T. Arslan, in Proceedings of the IEEE Antennas and Propagation Conference on Uniform Circular Arrays for Phased Array Antenna (Loughborough, UK, 2011)

14. K.R. Mahmoud, M.I. Eladawy, R. Bansal, S.H. Zainud-Deen, S.M. Ibrahem, Analysis of uniform circular arrays for adaptive beamforming applications using particle swarm optimization algorithm. Int. J. RF Microw. Comput. Aided Eng. 18(1), 42-52 (2007)

15. M.S. Hossain, G.N. Milford, M.C. Reed, in Proceedings of the IEEE International Symposium on Communication Information Technology (ISCIT) Efficient Robust Broadband Beamforming Using Circular Antenna Arrays (Gold Coast, Australia, 2012)

16. M. Askari, M. Karimi, in Proceedings of the IEEE Iranian Conference on Electrical Engineering Sector Beamforming with Uniform Circular Array Antennas Using Phase Mode Transformation (Mashhad, Iran, 2013)

17. T.A. Thomas, F.W. Vook, in Proceedings of the IEEE Wireless Commun. Networking Conference on Improving the Performance of the UCA When LTE Codebok Feedback (Shanghai, China, 2012)

18. L. Wu, H. Yang, D. Wang, in Proceedings of the IEEE Vehicular Technology Conference (VTC-Fall) Hadamard Transform Based Codebook Design for Uniform Circular Arrays in Mobile Radio Communications (Quebec City, QC, 2012)

19. A. Koc, A. Masmoudi, T. Le-Ngoc, in Proceedings of the IEEE Canadian Conference of Electrical and Computer Engineering (CCECE) on Hybrid Beamforming for Uniform Circular Arrays in Multi-user Massive MIMO Systems (Edmonton, Canada, 2019)

20. F. Zhang, W. Fan, Near-field ultra-wideband mmWave channel characterization using successive cancellation beamspace UCA algorithm. IEEE Trans. Veh. Technol. 68(8), 7248-7259 (2019)

21. R. Chen, R. Yao, W.X. Long, M. Moretti, J. Li, UCA-based OAM non-orthogonal multi-mode multiplexing. IEEE Open J. Antennas Propag. 2, 181-190 (2021) 
22. G.T. Gil, J.Y. Lee, H. Kim, D.H. Cho, Comparison of UCA-OAM and UCA-MIMO systems for sub-THz band line-of-sight spatial multiplexing transmission. J. Commun. Netw. 23(2), 83-90 (2021)

23. Y. Zeng, Y. Wang, Z. Chen, J. Zhang, J. Zhang, in Proceedings of the 202014 th European Conference on Antennas and Propagation (EUCAP) for Two-Dimensional OAM Radar Imaging Using Uniform Circular Antenna Arrays (Copenhagen, Denmark, 2020)

24. H. Jing, W. Cheng, X. Xia, A simple channel independent beamforming scheme with parallel uniform circular array. IEEE Commun. Lett. 23(3), 414-417 (2019)

25. D. Hu, Y. Zhang, L. He, J. Wu, Low-complexity deep-learning-based DOA estimation for hybrid massive MIMO systems with uniform circular arrays. IEEE Wirel. Commun. Lett. 9(1), 83-86 (2020)

26. M. Mahmood, A. Koc, T. Le-Ngoc, in Proceedings of the 2020 IEEE Global Communications Conference on 2D Antenna Array Structures for Hybrid Massive MIMO Precoding (Taipei, Taiwan, 2020)

27. J. Suh, C. Kim, W. Sung, J. So, S.W. Heo, Construction of a generalized DFT codebook using channel-adaptive parameters. IEEE Commun. Lett. 21(1), 196-199 (2017)

28. T. Liang, M. Zhu, F. Pan, A DOA estimation method for uniform circular array based on virtual interpolation and subarray rotation. IEEE Access $\mathbf{9}, 116760-116767$ (2021)

29. Q. Zhang et al., in Proceedings of the 2021 IEEE Statistical Signal Processing Workshop (SSP). A Cyclostationarity Based Esprit Algorithm for DOA Estimation of Uniform Circular Array (Rio de Janeiro, Brazil, 2021)

30. D. He, X. Chen, L. Pei, F. Zhu, L. Jiang, W. Yu, Multi-BS spatial spectrum fusion for 2-D DOA estimation and localization using UCA in massive MIMO system. IEEE Trans. Instrum. Meas. 70, 1-13 (2021)

31. R. Okada, K. Nishimori, in Proceedings of the 2021 International Symposium on Antennas and Propagation (ISAP). Circular Antenna Arrangement for LOS-MIMO Transmission Independent for the Transmission Distance Using a Genetic Algorithm (Osaka, Japan, 2021)

32. S.G. Mallat, A theory for multiresolution signal decomposition: the wavelet representation. IEEE Trans. Pattern Anal. Mach. Intell. 11(7), 674-693 (1989)

33. E. Guariglia, S. Silverstrov, Fractional-wavelet analysis of positive definite distributions and wavelets on $\mathcal{D}^{\prime}(\mathbb{C})$. Engineering Mathematics II, Springer Proceedings in Mathematics and Statistics, vol. 179 (Springer, 2017) 337-353

34. H.G.E. Hentschel, I. Procaccia, The infinite number of generalized dimensions of fractals and strange attractors. Phys. D 8(3), 435-444 (1983)

35. E. Guariglia, Harmonic Sierpinski gasket and applications. Entropy 20(9), 714 (2018)

36. S.G. Mallat, Multifrequency channel decompositions of images and wavelet models. IEEE Trans. Acoust. Speech Signal Process. 37(12), 2091-2110 (1989)

37. E. Guariglia, Primality, fractality and image analysis. Entropy 21(3), 304 (2019)

38. L. Yang, H. Su, C. Zhong, Z. Meng, H. Luo, X. Li, Y.Y. Tang, Y. Lu, Hyperspectral image classification using wavelet transform-based smooth ordering. Int. J. Wavelets Multiresol. Inf. Process. 17(6) Article Number 1950050 (2019)

39. E. Guariglia, Entropy and fractal antennas. Entropy 18(3), 84 (2016)

40. C.A. Balanis, Antenna Theory Analysis and Design, 3rd edn. (Wiley, NJ, 1997)

41. 3GPP TR 36.873, Study on 3D Channel Model for LTE, V12.7.0 (2018)

\section{Publisher's Note}

Springer Nature remains neutral with regard to jurisdictional claims in published maps and institutional affiliations.

\section{Submit your manuscript to a SpringerOpen ${ }^{\circ}$ journal and benefit from:}

- Convenient online submission

- Rigorous peer review

- Open access: articles freely available online

- High visibility within the field

- Retaining the copyright to your article

Submit your next manuscript at $\boldsymbol{\nabla}$ springeropen.com 